\title{
Estudio y análisis de conductas violentas en Educación Secundaria en España*
}

\author{
Study and Analysis of Violent Behaviour \\ in Secondary Education in Spain
}

Recibido: febrero 10 de 2009 | Revisado: agosto 10 de 2009 | Aceptado: septiembre 3 de 2009

\author{
José Jesús GÁzQuez ** \\ Mª Del CARMEn PÉREZ-Fuentes \\ JOSÉ J. CARRIÓN \\ Universidad de Almería, España \\ VÍCTOR SANTIUSTE ${ }^{* * *}$ \\ Universidad Complutense de Madrid, España
}

Para citar este artículo. Gázquez, J.J., Pérez-Fuentes, M.C., Carrión, J.J. \& Santiuste, V. (2010). Estudio y análisis de conductas violentas en Educación Secundaria en España. Universitas Psychologica, 9 (2), 371-380.

* Artículo de investigación.

** Facultad de Psicología, Departamento de Psicología Evolutiva y de la Educación. Ctra Sacramento s/n 04120 Almería, España. Correos electrónicos: jlinares@ual.es; sej473@ual.es

**** Facultad de Educación. Correo electrónico: victor. santiuste@edu.ucm.es

\section{RES U MEN}

El objetivo de este estudio, es comparar la prevalencia de determinadas conductas violentas en los mismos niveles educativos durante dos estudios transversales: el primero, realizado en el curso académico 2004-2005, y el segundo, tres años más tarde, durante el periodo 2007-2008. Para ello, se ha analizado una muestra total de 1.475 sujetos de edades comprendidas entre los 14 y 16 años. Los resultados muestran, que se produce un descenso significativo en la prevalencia de prácticamente todas las conductas violentas analizadas, tales como: insultos, peleas, etc., durante los años académicos mencionados anteriormente. Por otra parte, las mujeres son las que más sufren la agresión verbal indirecta y, por el contrario, los hombres son los que más ejercen este tipo de conducta.

Palabras clave autores

Violencia escolar, educación secundaria, prevalencia, estudiantes.

Palabras clave descriptores

Violencia en la educación, educación secundaria, agresividad en la adolescencia (psicología), España.

\section{A B S T R A C T}

The goal of this study was to compare prevalence of certain violent behaviors at the same educational level in two cross-sectional studies; the first one, carried out during the 2004-2005 academic course, and the second one three years later, during the 2007-2008 academic course. For this purpose, a total sample of 1.475 subjects, between 14 and 16 years of age, was analyzed. The results show that there is a significant decrease in the prevalence of almost all the violent behaviors analyzed, such as: insults, fights, etc., during the above-mentioned academic courses. However, the females suffer more indirect verbal aggression and, in contrast, the males perform this kind of behavior more frequently.

Key words authors

School Violence, Secondary Education, Prevalence, Students.

Key words plus

Violence in Education, Education, Secondary, Aggressiveness in Adolescence, Spain. 
Los sistemas educativos han asistido a un aumento progresivo del número de conflictos que se producen en los centros docentes, lo que supone un deterioro del clima de convivencia escolar y un argumento presente en cualquier reflexión acerca de la calidad educativa. Con la finalidad de conocer la prevalencia y la naturaleza de este tipo de problemas, se ponen en marcha múltiples investigaciones iniciadas por Heinemman en 1972. Desde entonces, los diversos estudios realizados en los últimos años, informan de la existencia de violencia o conflicto entre los escolares, en numerosos países, con un porcentaje no despreciable de estudiantes, alrededor del 15\%, que han sido intimidados o agredidos alguna vez o han presenciado alguna situación de conflicto (Debardieux \& Blaya, 2001; Smith, Morita, Junger-Tas, Olweus, Catalana \& Slee, 1999), también constatan la presencia de este tipo de problemas en toda clase de escuelas (Smith et al., 1999), analizan las relaciones de estas conductas con otros aspectos como el autoconcepto o la autoestima (De la Torre, García, Carpio \& Casanova, 2008; Estévez, Martínez \& Musitu, 2006), los comportamientos prosociales (Longobardi, Pasta \& Sclavo, 2008), así como los aspectos relacionados con su origen (Gázquez, Pérez, Lucas \& Palenzuela, 2008; Pelegrín \& Garcés de los Fayos, 2008; Yuste \& Pérez, 2008), los agentes implicados en estas conductas (profesores, familia y estudiantes) (Gázquez, Cangas, Pérez \& Lucas, 2008), y sus consecuencias (Hunter, MoraMerchán \& Ortega, 2004; Newman, Holden \& Delville, 2005).

Una de las pruebas indicativas de dicho incremento es la puesta en marcha de numerosas medidas políticas, así como el fomento de programas preventivos y de tratamiento para acabar con los aspectos que producen y deterioran la convivencia escolar (Garaigordobil, 2004; García, 2001). Este incremento también fue percibido por el profesorado, así, el 45\% de éste, en el año 1999, considera que la violencia ha aumentado ligeramente en su centro y un $32 \%$ considera que el aumento se ha producido de forma drástica (Defensor del Pueblo, 2000). Años más tarde, en 2006, el porcentaje de docentes que afirman que los conflictos han aumentado ligera o drásticamente es semejante al de 1999, aspecto que debe ser interpretado de forma prudente y puede poseer dos connotaciones diferentes, ya que por un lado, al no fijar el periodo temporal por el que se les cuestiona, su opinión podría verse influida o referida al previamente indicado en el año 1999; y, por el otro, podrían ser interpretadas las escasas diferencias en el porcentaje entre el año 1999 y 2006 como que realmente no se ha producido un aumento significativo de los conflictos (Cangas, Gázquez, Pérez, Padilla \& Miras, 2007; Defensor del Pueblo, 2007).

Esta última hipótesis se corresponde con las conclusiones obtenidas con el estudio comparativo de ambos informes. Así, en España, este último informe, indica que la incidencia del maltrato ha tendido claramente a disminuir, especialmente en las conductas más frecuentes y menos graves. Por ejemplo, los insultos pasan del $39,1 \%$ al $27 \%$ y la utilización de motes ofensivos lo hace del 37,7\% al 26,6\%. También disminuyen la agresión física indirecta, las amenazas, o el acoso sexual; pero, por el contrario, no muestran una tendencia a la baja y se mantienen en porcentajes parecidos a 1999 , aunque tampoco los superan, conductas como: la exclusión social, la agresión física o las formas de amenaza más graves (Defensor del Pueblo, 2007; Gázquez et al., 2008; Gázquez, Cangas, Pérez, Padilla \& Cano, 2007).

Otros estudios realizados, en diferentes ciudades españolas, nos hablan también de los tipos de conflicto que se producen en los centros españoles de Educación Secundaria. Así, por ejemplo, en Valladolid, el estudio realizado por Avilés y Monjas (2005), con una muestra de 496 alumnos entre 12 y 16 años pertenecientes a varios IES, nos indica que, la percepción del alumnado sobre los tipos de maltrato o intimidación existente en su centro, en términos de porcentajes, es la siguiente: insultar o poner motes $(43 \%)$, reírse o dejar en ridículo a otros $(34 \%)$, hacer daño físico (26\%), rechazar, aislar o no dejar participar (24\%), amenazar o chantajear para obligar a hacer algo al otro (22\%), hablar mal de alguien (12\%). Cuando en este mismo trabajo se les pregunta por su opinión personal sobre la forma más frecuente de maltrato entre 
compañeros, en primer lugar destacan la intimidación verbal, seguida de reírse de alguien o dejarlo en ridículo, en tercer lugar estaría el maltrato físico, seguido del aislamiento social y las amenazas en cuarto y quinto lugar, respectivamente, y en sexto y último lugar, encontramos hablar mal de alguien.

En Inglaterra, las modalidades de maltrato más frecuentes sufridas por los chicos y chicas de 12 a 16 años, fueron: Insultos, motes: 62\%; Agresión física: 26\%; Amenazas: 25\%; Rumores: 24\%; Insultos racistas: 9\% y Aislamiento social: 7\% (Whitney \& Smith, 1993). En Portugal, la violencia verbal directa es la más usual, tanto para chicos como para chicas, pero los chicos muestran consistentemente más agresión física y las chicas más agresión indirecta (Pereira, Mendoça, Neto, Almeida, Valente \& Smith, 1996). En Alemania el tipo de abusos que predominan de forma clara son los que implican agresión verbal y aquellos que conllevan violencia física leve.

En las escuelas elementales de Holanda, el estudio de Fekkes, Pijpers y Verloove-Vanhorick (2005), nos muestra los tipos de bullying o violencia escolar que los alumnos han experimentado durante las cuatro semanas anteriores a la fecha en que se realiza dicho estudio. De ello se desprende que el 30,9\% había sufrido insultos, el 24,8\% fue víctima de la expansión de diferentes tipos de rumores sobre ellos, el 17,2\% había sido ignorado o no lo habían dejado participar en alguna actividad, y el $14,7 \%$ declaraba haber recibido patadas o haber sido empujado. De todos los chicos que habían contestado haber experimentado bullying prácticamente a diario $(7,7 \%)$, el porcentaje declarado del tipo de conducta violenta sufrida es el siguiente: había recibido insultos el 90,3\%, habían sido víctimas de la extensión de rumores sobre ellos el 89,1\%, el 74,3\% había sufrido burlas, el 63,1\% había recibido patadas, golpes o empujones, el $60,8 \%$ había sido ignorado o no lo habían dejado participar en alguna actividad, y, por último, el $37,3 \%$ declaran que les han quitado o escondido alguna cosa.

Numerosos estudios hablan de un maltrato o violencia femenina de naturaleza distinta a la masculina (Barrio, Martín, Montero, Gutiérrez \&
Fernández, 2003; Underwood, Galen \& Paquette, 2001). De todos ellos se desprende que la agresión de tipo verbal indirecta, consistente en hablar mal de un tercero a sus espaldas, es más frecuente entre las chicas que entre los chicos, tanto a la hora de realizarlo como de sufrirlo, lo que no quiere decir que este tipo de agresión indirecta no se produzca también entre los chicos, aunque sí lo hacen con menor prevalencia. Sin embargo, estas diferencias no siempre son significativas (Avilés \& Monjas, 2005).

Otro fenómeno invisible incluido en el concepto de bullying y que afecta a la convivencia en los centros escolares, es el acoso sexual. Así por ejemplo, en Alemania (Funk, 1997) un 4\% de los chicos admiten haber sufrido acoso sexual por parte de sus compañeros. Mientras que en Holanda este porcentaje aumenta, ya que el $22 \%$ de las chicas holandesas admiten haber sufrido acoso sexual por parte de sus compañeros (Mooij, 1997). En España, encontramos un porcentaje inferior a ambos países, puesto que sólo un 0,9\% de los alumnos que cursan Educación Secundaria Obligatoria (mismo nivel que los estudios citados con anterioridad) declaran haber sido acosados sexualmente por alguno de sus compañeros (Defensor del Pueblo, 2007).

Todas estas cifras debemos observarlas con cautela y prudencia, ya que es difícil la comparación, entre los estudios realizados en los diversos países sobre los tipos de agresiones o modalidades de maltrato que se producen en las escuelas, debido a la utilización de definiciones y cuestionarios diferentes (Crothers \& Levinson, 2004; Defensor del Pueblo, 2007). Añadiendo otro aspecto por tener en cuenta y es cuando nos referimos y utilizamos el término de incidencia, y es que en la mayoría de los estudios, que apuntan hacia el aumento o descenso de la incidencia de las conductas violentas, no utilizan los mismos sujetos en los diferentes momentos temporales en los que se realizan los estudios, tratándose en todo caso de dos estudios de carácter transversal, en lugar de utilizar un diseño longitudinal con los mismos sujetos, siendo en este caso, cuando el término incidencia estaría bien empleado, pudiendo hablar, sólo en el resto de los casos, de prevalencia. 
El presente estudio, satisface las dos principales deficiencias de los estudios, ya que partimos de la misma definición de violencia escolar (Gázquez, Cangas, Padilla, Cano \& Pérez-Moreno, 2005) y se utiliza el mismo cuestionario en los dos momentos, en el estudio. Por otro lado, no se trata de un diseño longitudinal, sino que se plantean dos estudios transversales, cuyo objetivo es comparar los resultados de ambos estudios realizados, el primero de ellos, durante el curso académico 2004-2005, y el segundo, tres años más tarde, durante el curso académico 2007-2008. Por tanto, el objetivo de este estudio es comparar la prevalencia existente en diferentes cursos académicos de determinadas conductas violentas en los mismos niveles educativos.

\section{Método}

\section{Participantes}

La muestra está compuesta por un total de 1.475 sujetos, de los cuales 739 (51,1\%) son hombres y 734 (49,8\%) son mujeres. La edad de la muestra está comprendida entre los 14 y 16 años, con una media de 15,11 (DT =0,757). Así, del total de la muestra, el 23,8\% (351 sujetos) tienen 14 años, el $41,5 \%$ (612 sujetos) 15 años, y el 34,7\% (512 sujetos) tienen 16 años. Las características de la muestra, en función de los dos momentos temporales en los que se produce la aplicación del cuestionario, aparecen detalladas en la Tabla 1. Así, podemos observar que el Grupo 1 (alumnos/as del curso académico 2004-2005) se compone por un total de 849 sujetos, con una media de edad de 15,12 (DT $=0,756$ ), mientras que el Grupo 2 (alumnos/as del curso académico 2007-2008) está formado por un total de 626 sujetos, con una medida de edad de $15,09(\mathrm{DT}=0,756)$.

\section{Instrumentos}

Cuestionario para estudiantes (Ortega \& Del Rey, 2003), adaptado por Gázquez, Cangas, Padilla, Cano y Pérez-Moreno (2005).

Se trata de un cuestionario autoaplicado, en el que se indaga acerca de diferentes aspectos de la convivencia y la problemática de la violencia escolar, en los centros escolares. Para el presente estudio se han seleccionado los ítems que hacen referencia a: ¿Cuántas veces te sientes insultado/a, ridiculizado/a, te dicen motes y se meten verbalmente contigo, en el centro?; insultas a otro/a, lo/a ridiculizas, le dices motes y te metes verbalmente con él o ella?; ¿Te ha pasado que otros/as han hablado mal de ti a tus espaldas y has perdido los/as amigos/as por eso?; ¿Cuántas veces hablas mal de una persona que te cae mal, procurando que otros/ as también piensen mal de ella?; ¿Te has sentido

\section{TABLA 1}

Estadísticos descriptivos de la muestra en función del curso académico

\begin{tabular}{lccccccccc}
\hline & & \multicolumn{4}{c}{ Curso académico 2004-2005 } & \multicolumn{3}{c}{ Curso académico 2007-2008 } \\
\cline { 3 - 9 } & & 14 años & 15 años & 16 años & Total & 14 años & 15 años & 16 años & Total \\
\hline \multirow{2}{*}{ Hombre } & $\%$ & $11,5 \%$ & $20,8 \%$ & $18,8 \%$ & $51,2 \%$ & $8,8 \%$ & $24 \%$ & $15,8 \%$ & $48,6 \%$ \\
& $\mathrm{~N}$ & 98 & 177 & 160 & 435 & 55 & 150 & 99 & 304 \\
\multirow{2}{*}{ Mujer } & $\%$ & $11,5 \%$ & $20,5 \%$ & $16,5 \%$ & $48,5 \%$ & $16 \%$ & $17,7 \%$ & $17,7 \%$ & $51,4 \%$ \\
& $\mathrm{~N}$ & 98 & 174 & 140 & 412 & 100 & 111 & 111 & 322 \\
\multirow{2}{*}{ Total } & $\%$ & $23,1 \%$ & $41,3 \%$ & $35,6 \%$ & $100 \%$ & $24,8 \%$ & $41,7 \%$ & $33,5 \%$ & $100 \%$ \\
& $\mathrm{~N}$ & 196 & 351 & 302 & 849 & 155 & 261 & 210 & 626 \\
\hline
\end{tabular}

Fuente: elaboración propia. 
perseguido/a, hostigado/a o intimidado/a por otros/ as de forma prolongada?; ¿Has perseguido, hostigado e intimidado, en solitario o en grupo, a otros/as?; iTe has sentido alguna vez acosado/a sexualmente y has estado preocupado/a por esa razón?; y, ¿Crees que tu forma de expresarte y de comportarte ha podido dar lugar a que otro/a se sienta acosado/a sexualmente? Las diferentes cuestiones analizadas poseen cuatro alternativas de respuesta, así, las puntuaciones del cuestionario fueron transformadas y cuantificadas del siguiente modo: "Nunca" se corresponde con una puntuación de 0; "Alguna vez" con 1; "A veces" con 2; y por último, y finalmente "Muchas veces" con 3.

\section{Procedimiento}

El procedimiento utilizado es el comúnmente aceptado en Psicología Educativa (Delgado-Salazar \& Lara-Salcedo, 2008; Ison-Zintilini \& MorelatoGiménez, 2008). En un primer momento, se procedió a contactar con los directores de 10 centros educativos seleccionados de forma aleatoria, entre los centros de Educación Secundaria Obligatoria de la provincia de Almería para obtener su consentimiento. Una vez conseguido éste, se dio a conocer a los participantes (alumnos/as) la actividad a realizar y se solicitó su consentimiento, aplicándose posteriormente el cuestionario de forma colectiva en cada clase. La duración total de aplicación de la prueba fue aproximadamente de 15 minutos. Esta actuación se realizó durante el curso académico 2004-2005, y posteriormente en el curso académico 2007-2008. Para el análisis de los datos estadísticos fue utilizado el programa SPSS.15.

\section{Resultados}

Inicialmente se pretende conocer si existen diferencias significativas entre las puntuaciones medias de cada uno de los grupos. No se cumple la homocedasticidad de la muestra, salvo para el ítem ¿Cuántas veces te sientes insultado/a, ridiculizado/a, te dicen motes y se meten verbalmente contigo, en el centro?, donde la Prueba Le- vene $[F(1,1466)=2,384, p>0,05]$ nos muestra la homogeneidad de las varianzas, caso en el cual, se ha realizado la prueba ANOVA de un factor, encontrando diferencias significativas entre ambos grupos $[F(1,1466)=3,948, p<0,05]$, siendo la media de la muestra del Grupo 1 de 0,7 (DT = $0,827)$ y la del segundo de $0,62(D T=0,755)$, y mostrando una disminución significativa en los insultos, motes, etc. En el resto de los casos, donde no se cumple el criterio de homocedasticidad, se ha realizado la prueba $U$ de Mann-Whitney cuyos resultados aparecen en la Tabla 2. Así, podemos observar que en todos los casos se produce una disminución de carácter significativo, excepto para el ítem ¿Cuántas veces hablas mal de una persona que te cae mal, procurando que otros/as también piensen mal de ella?, donde se produce un pequeño descenso desde el curso académico 2004-2005 al 2007-2008, pero éste no es significativo.

Un análisis por género, nos muestra que, a la hora de realizar la comparación entre los dos grupos, en los hombres, se cumple la homocedasticidad de la muestra para los ítems: ¿Cuántas veces te sientes insultado/a, ridiculizado/a, te dicen motes y se meten verbalmente contigo, en el centro? [Prueba Levene $F(1,733)=1,957, p>0,05]$, realizando para conocer si existen diferencias significativas entre las puntuaciones medias de cada grupo la prueba ANOVA de un factor, encontrando diferencias significativas entre ambos grupos $[F(1,733)$ $=7,298, p<0,01]$, siendo la media de la muestra del grupo 1 de $0,79(D T=0,875)$ y la del segundo de $0,63(D T=0,738)$; insultas a otro/a, lo/a ridiculizas, le dices motes y te metes verbalmente con él o ella? [Prueba Levene $F(1,733)=3,089$, $p>0,05]$, y la ANOVA de un factor nos muestra diferencias significativas entre ambos grupos $[F(1,733)=22,147, p<0,01]$, siendo la media de la muestra del Grupo 1 de 0,82 $(D T=0,856)$ y la del segundo de 0,54 (DT =0,712); ¿Cuántas veces hablas mal de una persona que te cae mal, procurando que otros/as también piensen mal de ella? [Prueba Levene $F(1,733)=2,879, p>0,05$ ], y la ANOVA de un factor nos señala que no existen diferencias significativas entre ambos grupos $(F(1,733)=0,586, p>0,05)$, siendo la media de 
TABLA 2

Diferencia de medias en los cursos académicos. Prueba U de Mann-Whitney

\begin{tabular}{|c|c|c|c|c|c|c|c|c|c|}
\hline & \multicolumn{3}{|c|}{$\begin{array}{l}\text { Curso académico } \\
\text { 2004-2005 }\end{array}$} & \multicolumn{3}{|c|}{$\begin{array}{l}\text { Curso académico } \\
2007-2008\end{array}$} & \multirow{2}{*}{$\begin{array}{l}\text { U de Mann- } \\
\text { Whitney }\end{array}$} & \multirow{2}{*}{$Z$} & \multirow{2}{*}{ Sig. } \\
\hline & $\mathrm{N}$ & Media & DT & $\mathrm{N}$ & Media & DT & & & \\
\hline $\begin{array}{l}\text { ¿Insultas a otro/a, lo/a ridiculizas, le } \\
\text { dices motes y te metes verbalmente } \\
\text { con él o ella? }\end{array}$ & 869 & 0,70 & 0,803 & 626 & 0,40 & 0,643 & 211133,500 & $-7,377$ &, 000 \\
\hline $\begin{array}{l}\text { ¿Te ha pasado que otros/as han ha- } \\
\text { blado mal de ti a tus espaldas y has } \\
\text { perdido los/as amigos/as por eso? }\end{array}$ & 866 & 0,71 & 0,770 & 626 & 0,53 & 0,676 & 227385,500 & $-4,881$ &, 000 \\
\hline $\begin{array}{l}\text { ¿Cuántas veces hablas mal de una } \\
\text { persona que te cae mal, procurando } \\
\text { que otros/as también piensen mal } \\
\text { de ella? }\end{array}$ & 869 & 0,68 & 0,810 & 626 & 0,60 & 0,638 & 263132,500 &,- 057 & ,954 \\
\hline $\begin{array}{l}\text { ¿Te has sentido perseguido/a, } \\
\text { hostigado/a o intimidado/a por } \\
\text { otros/as de forma prolongada? }\end{array}$ & 869 & 0,38 & 0,650 & 626 & 0,16 & 0,392 & 223675,500 & $-6,678$ &, 000 \\
\hline $\begin{array}{l}\text { ¿Has perseguido, hostigado e inti- } \\
\text { midado, en solitario o en grupo, a } \\
\text { otros/as? }\end{array}$ & 865 & 0,37 & 0,690 & 626 & 0,14 & 0,390 & 225393,000 & $-6,615$ &, 000 \\
\hline $\begin{array}{l}\text { ¿Te has sentido acosado/a sexual- } \\
\text { mente y has sentido miedo por esa } \\
\text { razón? }\end{array}$ & 867 & 0,23 & 0,674 & 626 & 0,10 & 0,434 & 242897,000 & $-4,681$ &, 000 \\
\hline $\begin{array}{l}\text { ¿Crees que tu forma de expresarte y } \\
\text { de comportarte ha podido dar lugar } \\
\text { a que otro/a se sienta acosado/a } \\
\text { sexualmente? }\end{array}$ & 866 & 0,22 & 0,616 & 626 & 0,09 & 0,441 & 239911,500 & $-5,420$ &, 000 \\
\hline
\end{tabular}

Fuente: elaboración propia.

la muestra del Grupo 1 de 0,67 (DT =0,821) y la del segundo de 0,62 (DT =0,693). Sólo en los dos primeros casos, los hombres están significativamente menos afectados y/o muestran menor prevalencia de esas conductas en el curso académico 2007-2008 que en el curso académico 2004-2005. En el resto de los casos, se realiza la prueba $U$ de Mann-Whitney cuyos resultados aparecen en la Tabla 3, donde podemos observar como en todos los casos se produce una disminución significativa de la prevalencia y/o afectación en los sujetos varones de esas conductas en el curso académico 2007-2008 respecto al curso 2004-2005.
Por otro lado, en las mujeres, se cumple la homocedasticidad de la muestra para los ítems: ¿Cuántas veces te sientes insultado/a, ridiculizado/a, te dicen motes y se meten verbalmente contigo, en el centro? [Prueba Levene $F(1,731)=0,017, p>0,05$ ], realizando para conocer si existen diferencias significativas entre las puntuaciones medias de cada grupo la ANOVA de un factor, encontrando diferencias significativas entre ambos grupos $(F(1,731)$ $=0,007, p>0,05)$ siendo la media de la muestra del Grupo 1 de 0,60 (DT = 0,753) y la del segundo de $0,61(D T=0,771)$; $y$ iTe ha pasado que otros/as han hablado mal de ti a tus espaldas y has perdido 
TABLA 3

Diferencia de medias en los hombres. Prueba U de Mann-Whitney

\begin{tabular}{|c|c|c|c|c|c|c|c|c|c|}
\hline & \multicolumn{3}{|c|}{$\begin{array}{l}\text { Curso académico } \\
2004-2005\end{array}$} & \multicolumn{3}{|c|}{$\begin{array}{c}\text { Curso académico } \\
2007-2008\end{array}$} & \multirow{2}{*}{$\begin{array}{l}\text { U de Mann- } \\
\text { Whitney }\end{array}$} & \multirow[t]{2}{*}{$\mathrm{Z}$} & \multirow[t]{2}{*}{ Sig. } \\
\hline & $\mathrm{N}$ & Media & DT & $\mathrm{N}$ & Media & DT & & & \\
\hline $\begin{array}{l}\text { ¿Te ha pasado que otros/as han ha- } \\
\text { blado mal de ti a tus espaldas y has } \\
\text { perdido los/as amigos/as por eso? }\end{array}$ & 430 & 0,64 & 0,768 & 304 & 0,46 & 0,654 & 57515,500 & $-3,126$ & ,002 \\
\hline $\begin{array}{l}\text { ¿Te has sentido perseguido/a, } \\
\text { hostigado/a o intimidado/a por } \\
\text { otros/as de forma prolongada? }\end{array}$ & 432 & 0,41 & 0,678 & 304 & 0,17 & 0,421 & 54520,000 & $-5,166$ & ,000 \\
\hline $\begin{array}{l}\text { ¿Has perseguido, hostigado e inti- } \\
\text { midado, en solitario o en grupo, a } \\
\text { otros/as? }\end{array}$ & 431 & 0,43 & 0,752 & 304 & 0,19 & 0,470 & 55631,000 & $-4,653$ & ,000 \\
\hline $\begin{array}{l}\text { ¿Te has sentido acosado/a sexual- } \\
\text { mente y has sentido miedo por esa } \\
\text { razón? }\end{array}$ & 430 & 0,28 & 0,787 & 304 & 0,10 & 0,397 & 60552,500 & $-3,124$ & ,002 \\
\hline $\begin{array}{l}\text { ¿Crees que tu forma de expresarte y } \\
\text { de comportarte ha podido dar lugar } \\
\text { a que otro/a se sienta acosado/a } \\
\text { sexualmente? }\end{array}$ & 429 & 0,29 & 0,685 & 304 & 0,11 & 0,481 & 57092,500 & $-4,820$ & ,000 \\
\hline
\end{tabular}

Fuente: elaboración propia.

los/as amigos/as por eso? [Prueba Levene $F(1,729)$ $=0,573, p>0,05]$ realizando para conocer si existen diferencias significativas entre las puntuaciones medias de cada grupo la ANOVA de un factor, encontrando diferencias significativas entre ambos grupos $(F(1,729)=14,794, p<0,01)$, siendo la media de la muestra del Grupo 1 de 0,80 (DT = $0,760)$ y la del segundo de $0,59(D T=0,692)$. Por lo tanto, aunque en los dos casos podamos hablar de un descenso en la prevalencia y/o afectación de los sujetos por esas conductas, sólo en el último de ellos, iTe ha pasado que otros/as han hablado mal de ti a tus espaldas y has perdido los/as amigos/as por eso?, las disminución es significativa. En los casos en los que no se cumple la homocedasticidad se realiza la prueba $U$ de Mann-Whitney cuyos resultados aparecen en la Tabla 4. Tal y como podemos observar, la diferencia de medias entre los dos grupos es significativa para todos los ítems, excepto el que hace referencia a ¿Cuántas veces hablas mal de una persona que te cae mal, procu- rando que otros/as también piensen mal de ella?, pudiendo indicar en este caso un ligero descenso de esta conducta pero no significativo en el año 2007-2008 respecto al 2004-2005. En el resto de los ítems, la disminución de las conductas entre las mujeres es significativa en el año académico 2007-2008 respecto al 2004-2005.

\section{Discusión}

Son varios los problemas o inconvenientes que nos encontramos cuando analizamos la violencia escolar, en primer lugar es la utilización del término incidencia, cuando realmente dichos trabajos lo que ponen de manifiesto es la prevalencia de determinadas conductas en distintos momentos, utilizando para ello la metodología transversal. Por otra parte, cuando comparamos los datos de diferentes estudios, nos encontramos que todos ellos emplean cuestionarios y definiciones distintas (Crothers \& Levinson, 2004; Defensor del Pueblo, 
TABLA 4

Diferencia de Medias en las mujeres. Prueba U de Mann-Whitney

\begin{tabular}{|c|c|c|c|c|c|c|c|c|c|}
\hline & \multicolumn{3}{|c|}{$\begin{array}{l}\text { Curso académico } \\
2004-2005\end{array}$} & \multicolumn{3}{|c|}{$\begin{array}{l}\text { Curso académico } \\
2007-2008\end{array}$} & \multirow{2}{*}{$\begin{array}{l}\text { U de Mann- } \\
\text { Whitney }\end{array}$} & \multirow{2}{*}{ Z } & \multirow{2}{*}{ Sig. } \\
\hline & $\mathrm{N}$ & Media & DT & $\mathrm{N}$ & Media & DT & & & \\
\hline $\begin{array}{l}\text { ¿Insultas a otro/a, lo/a ridiculizas, le } \\
\text { dices motes y te metes verbalmente } \\
\text { con él o ella? }\end{array}$ & 411 & 0,54 & 0,692 & 322 & 0,28 & 0,542 & 52407,000 & $-5,765$ & ,000 \\
\hline $\begin{array}{l}\text { ¿Cuántas veces hablas mal de una } \\
\text { persona que te cae mal, procurando } \\
\text { que otros/as también piensen mal } \\
\text { de ella? }\end{array}$ & 411 & 0,62 & 0,708 & 322 & 0,58 & 0,582 & 66091,500 &,- 031 & ,975 \\
\hline $\begin{array}{l}\text { ¿Te has sentido perseguido/a, } \\
\text { hostigado/a o intimidado/a por } \\
\text { otros/as de forma prolongada? }\end{array}$ & 410 & 0,33 & 0,582 & 322 & 0,16 & 0,363 & 57345,000 & $-4,204$ & ,000 \\
\hline $\begin{array}{l}\text { ¿Has perseguido, hostigado e inti- } \\
\text { midado, en solitario o en grupo, a } \\
\text { otros/as? }\end{array}$ & 407 & 0,27 & 0,575 & 322 & 0,09 & 0,287 & 57278,000 & $-4,617$ & ,000 \\
\hline $\begin{array}{l}\text { ¿Te has sentido acosado/a sexual- } \\
\text { mente y has sentido miedo por esa } \\
\text { razón? }\end{array}$ & 410 & 0,20 & 0,553 & 322 & 0,10 & 0,466 & 60809,000 & $-3,503$ & ,000 \\
\hline $\begin{array}{l}\text { ¿Crees que tu forma de expresarte y } \\
\text { de comportarte ha podido dar lugar } \\
\text { a que otro/a se sienta acosado/a } \\
\text { sexualmente? }\end{array}$ & 410 & 0,14 & 0,524 & 322 & 0,07 & 0,400 & 63042,000 & $-2,436$ & ,015 \\
\hline
\end{tabular}

Fuente: elaboración propia.

2007; Espelage \& Swearer, 2003; Griffin \& Gross, 2004; Solberg \& Olweus, 2003). A pesar de ello, son escasos los estudios en España que cumplen con las dos primeras características, como puede ser el estudio del Defensor del Pueblo (2007) y el que aquí se describe, desprendiéndose de ambos un descenso en la prevalencia de las conductas violentas.

Así, es significativo el descenso observado respecto a los insultos, conductas de ridiculización, tanto recibidas como realizadas, así como otros tipos de conductas, como por ejemplo: que otros hablen mal de ti a tus espaldas, sentirse perseguido, hostigado o intimidado por otros de forma prolongada, sentirse acosado sexualmente, o considerar que tu forma de expresarte y comportarte ha podido generar en otros el sentimiento de acoso.
Resultados éstos, que coinciden con los indicados por el Defensor del Pueblo (2007) donde se destaca, sobre todo, la disminución de la agresión física indirecta, las amenazas, y el acoso sexual.

En el análisis realizado teniendo en cuenta el género, el presente trabajo está en sintonía con numerosos estudios realizados y se constata que la agresión de tipo verbal indirecta (hablar de otro mal a sus espaldas), es más frecuente entre las chicas que entre los chicos, tanto a la hora de realizarlo como de sufrirlo (Avilés \& Monjas, 2005; Barrio et al., 2003; Underwood et al., 2001), aunque pudiendo matizar, que en el presente estudio, la prevalencia de este tipo de conducta es mayor por parte del género femenino que lo sufre, mientras que en el masculino son más los que la ejercen o realizan. 
Teniendo en cuenta, que el impacto de sufrir este tipo de violencia no es el mismo en todas las personas, ya que según Newman, Holden y Delville (2005), esto depende del grado en que la persona sienta ese aislamiento, pero que tal y como muestran los diferentes estudios, esta huella se produce en la mayoría de los casos y tiene repercusión sobre el sujeto, mostrando índices más altos (Newman, Holden \& Delville, 2005), teniendo en cuenta, además, que las víctimas que declaran una baja percepción de control sobre el bullying y que valoran la situación como una amenaza, presentan aún mayores niveles de angustia que aquellos que sienten un mayor control y que valoran dicha situación como un reto que han de afrontar (Hunter, Mora-Merchán \& Ortega, 2004). El siguiente paso será proponer estrategias de intervención (Garaigordobil, 2004) para disminuir la prevalencia de aquellas conductas violentas que en el presente estudio no han mostrado una disminución significativa, como es la presencia de la violencia verbal e indirecta, hablar mal de una persona con la intención de influir en los otros en su contra. Así, a través de la intervención en este aspecto, es también probable que se alcance una menor prevalencia en la presencia del resto de las conductas analizadas y que forman parte de los actos o conductas de violencia, dentro de la escuela.

\section{Referencias}

Avilés, J. M. \& Monjas, I. (2005). Estudio de incidencia de la intimidación y el maltrato entre iguales en la educación secundaria obligatoria mediante el cuestionario CIMEI: Cuestionario sobre Intimidación y Maltrato entre Iguales. Anales de Psicología, 21(1), 27-41.

Barrio, C., Martín, E., Montero, I., Gutiérrez, H. \& Fernández, I. (2003). La realidad del maltrato entre iguales en los centros de secundaria españoles. Infancia y Aprendizaje, 26(1), 25-47.

Cangas, A. J., Gázquez, J. J., Pérez-Fuentes, M. C., Padilla, D. \& Miras, F. (2007). Evaluación de la violencia escolar y su afectación personal en una muestra de estudiantes europeos. Psicothema, 19(1), 114-119.
Crothers, L. M. \& Levinson, E. M. (2004). Assessment of bullying: A review of methods and instruments. Journal of Counseling and Development, 82, 496503.

Debardieux, E. \& Blaya, C. (2001). La violence en milieu scolaire. Dix aproches en Europe. París: ESF.

De La Torre, M. J., García, M. C., Carpio, M. V. \& Casanova, P. F. (2008). Relaciones entre violencia escolar y autoconcepto multidimensional en adolescentes de Educación Secundaria Obligatoria. European Journal of Education and Psychology, 1(2), 57-70.

Delgado-Salazar, R. \& Lara-Salcedo, L. M. (2008). De la mediación del conflicto escolar a la construcción de comunidades justas. Universitas Psychologica, 7(3), 673-690.

España, Defensor del Pueblo (2000). Informe sobre violencia escolar: el maltrato entre iguales en la educación secundaria obligatoria. Madrid: Autor.

España, Defensor del Pueblo (2007). Violencia Escolar: El maltrato entre iguales en la Educación Secundaria Obligatoria 1999-2006. Madrid: Autor.

Espelage, D. L. \& Swearer, S. M. (2003). Research on school bullying and victimizacion: What have we learned and were do we go from here? School Psychology Review, 32 (3), 365-383.

Estévez, E., Martínez, B. \& Musitu, G. (2006). La autoestima en agresores y víctimas en la escuela: la perspectiva multidimensional. Intervención Psicosocial, 15, 223-232.

Fekkes, M., Pijpers, F. I. M. \& Verloove-Vanhorick, S. P. (2005). Bullying: Who does what, when and where? Involvement of children, teachers and parents in bullying behaviour. Health Education Research, 20(1), 81-91.

Funk, W. (1997). Violencia escolar en Alemania. Revista de Educación, 313, 53-78.

Garaigordobil, M. (2004). Intervención psicológica en la conducta agresiva y antisocial con niños. Psicothema, 16(3), 429-235.

García, A. (2001). La violencia en centros educativos de Norteamérica y diferentes países de Europa. Medidas políticas tomadas para la convivencia escolar. Revista Interuniversitaria de Formación del Profesorado, 41, 167-177. 
Gázquez, J. J., Cangas, A. J., Padilla, D., Cano, A. \& Pérez-Moreno, P. J. (2005). Assessment by pupils, teachers and parents of school coexistence problems in Spain, France, Rep. Checa and Hungary: Global psychometric data. International Journal of Psychology and Psychological Therapy, 5(2), 101 . 112.

Gázquez, J. J., Cangas, A. J., Pérez-Fuentes, M. C. \& Lucas, F. (2008). Comparative analysis of the perception of school violence in teachers, pupils and families. The Spanish Journal of Psychology, $11(2), 443-452$.

Gázquez, J. J., Cangas, A. J., Pérez-Fuentes, M. C., Padilla, D. \& Cano, A. (2007). Percepción de la violencia escolar por parte de los familiares: un estudio comparativo en cuatro países europeos. International Journal of Clinical and Health Psycho$\log$, , 7(1), 93-105.

Gázquez, J. J., Pérez-Fuentes, M. C., Lucas, F. \& Palenzuela, M. M. (2008). Percepción del alumnado universitario sobre el origen de la violencia escolar. European Journal of Education and Psychology, $1(1), 68-80$.

Griffin, R. S. \& Gross, A. M. (2004). Childhood bullying: Current empirical findings and future directions for research. Aggression and Violent Behavior, 9, 379-400.

Heinemman, P. P. (1972). Mobbing-gruppvald blant barn och vuxna [Violencia grupal y mobbing entre niños y adultos]. Stockholm: Naturoch Kultur.

Hunter, S. C., Mora-Merchán, J. \& Ortega, R. (2004). The long-term effects of doping strategy use in victims of bullying. The Spanish Journal of Psycho$\log y, 7,3-12$.

Ison-Zintilini, M. S. \& Morelato-Giménez, G. S. (2008). Habilidades socio-cognitivas en niños con conductas disruptivas y víctimas de maltrato. Universitas Psychologica, 7(2), 357-367.
Longobardi, C., Pasta, T. \& Sclavo, E. (2008). The educative relationship in primary school: Aggressive tendencies and pro-social behavior. European Journal of Education and Psychology, 1(2), 5-18.

Mooij, T. (1997). Por la seguridad en la escuela. Revista de Educación, 313, 29-52.

Newman, M. L., Holden, G. W. \& Delville, Y. (2005). Isolation and the stress of being bullied. Journal of Adolescence, 28, 343-357.

Ortega, R. \& Del Rey, R. (2003). La violencia escolar: estrategias de prevención. Barcelona: Graó.

Pelegrín, A. \& Garcés de Los Fayos, E. J. (2008). Variables contextuales y personales que inciden en el comportamiento violento del niño. European Journal of Education and Psychology, 1(1), 5-20.

Pereira, B., Mendoça, D. Neto, C., Almeida, A. Valente, L. \& Smith, P. K. (1996, agosto). Facts and figures of the first survey on bullying in Portuguese schools. Comunicación presentada en European Conference on Educational Research, Sevilla, España.

Smith, P. K., Morita, Y., Junger-Tas, J., Olweus, D., Catalano, R. \& Slee, P. (1999). The nature of school bullying. A cross-national perspectiva. Londres: Routledge.

Solberg, M. E. \& Olweus, D. (2003). Prevalence estimation of school bullying with the Olweus Bully/ Victim Questionnaire. Aggressive Behavior, 29, 239-268.

Underwood, M. K., Galen, B. R. \& Paquette, J. A. (2001). Top ten challenges for understanding gender and aggression in children: Why can't we all just get along? Social Development, 10, 248-266.

Whitney, I. \& Smith, P. K. (1993). A survey of the nature and extent of bullying in junior middle and secondary schools. Educational Research, 35, 3-25.

Yuste, N. \& Pérez, M. C. (2008). Las cuestiones familiares como causa de la violencia escolar según los padres. European Journal of Education and Psychology, 1(2), 19-27. 